\title{
Clonidine Blocks Acquisition But not Expression of Conditioned Opiate Withdrawal in Rats
}

\author{
Gery Schulteis, Ph.D., Luis Stinus, Ph.D., Victoria B. Risbrough, B.A., and George F. Koob, Ph.D.
}

\begin{abstract}
Previous studies in rodents have reported that clonidine, an $\alpha_{2}$-adrenergic receptor agonist, attenuated conditioned aversions to naloxone-precipitated opiate withdrawal when administered prior to each withdrawal conditioning episode. The current study was designed to determine whether clonidine could modify the expression of previously established conditioned place aversions and conditioned suppression of operant responding. Dose- and time-dependent effects of clonidine on activity and suppression of operant responding for food identified appropriate treatment parameters for subsequent studies in which rats rendered dependent on opiates through implantation of morphine pellets were tested for: (1)
\end{abstract}

conditioned place aversion; and (2) conditioned suppression of operant responding for food (fixed ratio-15 schedule), in a paradigm wherein rats received four pairings of naloxone with a distinct tone and odor stimulus. Clonidine dosedependently blocked the acquisition of both conditioned behaviors when administered prior to naloxone on each conditioning trial, but was ineffective in blocking the expression of these conditioned withdrawal signs when administered prior to the test session.

[Neuropsychopharmacology 19:406-416, 1998] (c) 1998 American College of Neuropsychopharmacology. Published by Elsevier Science Inc.
KEY WORDS: Clonidine; Opiate dependence; Opiate withdrawal; Conditioning; Naloxone

Addiction can be defined as a "behavioral pattern of drug use, characterized by overwhelming involvement with the use of a drug (compulsive use), the securing of its supply, and a high tendency to relapse after withdrawal" (Jaffe 1990). Among the factors that may contribute to loss of control over drug intake and relapse

From the Department of Neuropharmacology (GS, VBR, GFK), The Scripps Research Institute, La Jolla; Department of Anesthesiology (GS), U.C. San Diego School of Medicine, San Diego, California, USA; and Laboratoire de Neuropsychobiologie des Desadaptations (LS), Université de Bordeaux II, Bordeaux, Cedex, France.

Address correspondence to: G. Schulteis, Department of Anesthesiology, VAMC 125, U.C. San Diego School of Medicine, $3350 \mathrm{La}$ Jolla Village Drive, San Diego, CA 92161-5085, USA.

Received November 21, 1997; revised February 9, 1998; accepted February 16, 1998. after periods of abstinence are conditioned associations that are formed over a course of repeated drug experience. Within the domain of opiate withdrawal symptomatology, it is now recognized that somatic/autonomic signs and affective or emotional signs (e.g., anxiety, depression, dysphoria), each with their own unique underlying neurophysiological substrates (reviewed by Maldonado et al. 1996; Schulteis and Koob 1996), may differentially motivate continued drug use, with the affective signs hypothesized to be of greater motivational significance than the somatic signs.

A considerable body of both clinical and preclinical literature suggests that positive (drug reward) and negative (drug withdrawal) affective states can become associated with stimuli in the drug-taking environment and that these conditioned stimuli themselves acquire motivational significance in maintaining compulsive use and in precipitating relapse after periods of abstention (Baldwin and Koob 1993; Childress et al. 1994; O'Brien 
et al. 1976, 1993; Ramsay and Woods 1997; Schulteis and Koob 1996; Schulteis et al. 1997a; Wikler 1973).

Successful implementation of treatment strategies that will facilitate detoxification and reduce the probability of relapse must recognize the individual elements that contribute to the addiction process and the degree to which a given treatment strategy is able to address those individual elements, both unconditioned and conditioned. For example, clonidine, a noradrenergic alpha $_{2}$ agonist, has been found in both clinical (Charney et al. 1981; Cuthill et al. 1990; Dawe and Gray 1995; Gold et al. 1978; Janiri et al. 1994; Jasinski et al. 1985; Kasvikis et al. 1990) and preclinical animal studies (Coupar 1992; Katz 1986; Kelsey et al. 1990; Sparber and Meyer 1978; Taylor et al. 1988) to ameliorate some of the effects of opiate withdrawal. Treatment strategies based upon clonidine therapy are aimed primarily at facilitating opiate detoxification through suppression of somatic opiate withdrawal symptoms. However, in many of these same studies, it was reported that subjective signs of such withdrawal discomfort as dysphoria, anxiety, irritability, restlessness, and drug craving were attenuated only partially, or not at all, when clonidine was substituted for opiates in addicts. Moreover, there are reports that addicts will relapse to drug intake even during the period of clonidine therapy (Cuthill et al. 1990). Therefore, based upon the existing clinical literature, it seems apparent that there are potentially significant limitations to the efficacy of clonidine therapy to facilitate detoxification, to suppress withdrawal, and to decrease the probability of relapse to opiate use. Data from the preclinical literature may provide some clues to understanding these limitations.

Preclinical studies have verified the efficacy of clonidine in blocking a number of somatic and autonomic signs produced by spontaneous or naloxone-precipitated opiate withdrawal in rodents and monkeys (Britton et al., 1984; Coupar 1992; Kantak and Miczek 1988; Katz 1986; Sparber and Meyer 1978; Taylor et al. 1988). In addition, recent studies with conditioned place aversion, a behavioral paradigm that serves as a sensitive animal model of affective opiate withdrawal (Hand et al. 1988; Schulteis et al. 1994), suggested that clonidine administered prior to each conditioning trial could block the acquisition of a conditioned aversion to a distinct environment paired with naloxone-precipitated withdrawal (Kosten 1994; Nader and van der Kooy 1996). Conditioned place aversions can be elicited by administration of opiate antagonists directly into the nucleus accumbens and central nucleus of the amygdala (Stinus et al. 1990), critical elements of the brain's reward circuitry that mediate the acute hedonic (rewarding) effects of opiates and other drugs of abuse (see Schulteis and Koob 1996; Schulteis et al. 1997a). Therefore, conditioned place aversions seem to be a specific manifestation of neuroadaptation within the affective (emotional) domain. Accordingly, results indicating that clonidine could prevent the acquisition of conditioned place aversions were interpreted as suggesting that affective components of opiate withdrawal were sensitive to clonidine, at least under some conditions (Kosten 1994).

Untested, however, was whether clonidine administered once the conditioned aversion was established could prevent the expression of conditioned withdrawal. This situation would more closely approximate the clinical condition wherein any conditioned associations between opiate reinforcement or opiate withdrawal and environmental stimuli would have been formed long before onset of clonidine therapy. Therefore, the current set of studies was undertaken to examine the relative ability of clonidine to attenuate the acquisition and expression of conditioned opiate withdrawal. In addition to employing conditioned place aversions as an index of conditioned affective opiate withdrawal, we also included conditioned suppression of operant responding for food (Baldwin and Koob 1993). Suppression of operant responding for food is a sensitive index of antagonist precipitated opiate withdrawal that is attenuated by clonidine pretreatment (Sparber and Meyer 1978). Its validity as an animal model of affective withdrawal again is supported by the ability to precipitate suppression of operant responding by direct application of opiate antagonists to the nucleus accumbens (Koob et al. 1989; see Schulteis et al. 1997a for further discussion).

\section{GENERAL METHODS}

All procedures employed in the studies described herein were reviewed and approved by appropriate Institutional Animal Care and Use Committees, and were carried out in accordance with the guidelines established in the Guide for the Care and Use of Laboratory Animals (National Research Council and National Institutes of Health).

\section{Subjects}

For studies conducted at INSERM Unit 259 in Bordeaux, France (Experiments 1 and 4), male SpragueDawley rats (IFFA CREDO, Lyon, France) weighing 280 to $300 \mathrm{~g}$ at the beginning of the experiment were used. For studies conducted at The Scripps Research Institute in La Jolla, California (Experiments 2 and 3), male Wistar rats (Charles River, Kingston, NY) weighing 280 to $300 \mathrm{~g}$ at the start of each experiment were used. All rats were group housed (2-3/cage) in temperature- and humidity-controlled rooms with a 12-hour light/12-hour dark cycle. All rats except those trained to level press for 
food (Experiments 2 and 3) had ad libitum access to food, and all rats had ad libitum access to water. Rats trained to lever press for food were maintained on $15 \mathrm{~g}$ of rat chow per day in addition to the food pellets earned in the operant boxes (total food intake was approximately $22 \mathrm{~g} / \mathrm{rat} /$ day). All training and testing took place during the active (lights out) portion of the rats' daily activity cycle.

\section{Drugs}

Morphine pellets (75 mg base per pellet) were obtained from the National Institute on Drug Abuse (NIDA). Naloxone $\mathrm{HCl}$ and clonidine $\mathrm{HCl}$ were purchased from Sigma (St. Louis, MO) and dissolved in $0.9 \%$ saline for injection. All injections were made subcutaneously (SC) in a volume of $0.1 \mathrm{ml} / 100 \mathrm{~g}$ body weight.

Dependence Induction. Rats were made dependent on morphine through SC implantation of morphine pellets. The rats were anesthetized with halothane (1.5$2.0 \% \mathrm{v} / \mathrm{v}$ in air), and two pellets each containing $75 \mathrm{mg}$ of morphine base were implanted SC in the upper back. A minimum of 3 days elapsed between pellet implantation and any further behavioral testing of the animals. Earlier studies have shown stable plasma levels of morphine between 3 and 12 days after SC implant of two morphine pellets (Gold et al. 1994). Therefore, all behavioral testing in the current study was limited to 3 to 12 days postpellet implantation.

Spontaneous Locomotion in Photocell Cages. Locomotor activity was measured in 32 wire cages $(25 \mathrm{~cm}$ high $\times$ $25 \mathrm{~cm}$ wide $\times 36 \mathrm{~cm}$ long), each of which had two horizontal infrared beams across the long axis of the cage 3 $\mathrm{cm}$ above the floor and $14 \mathrm{~cm}$ apart. Total photocell beam interruptions were recorded by a computer in 10or 60-min intervals depending on the experiment.

Operant Responding for Food. As described in detail elsewhere (Schulteis et al. 1994), rats initially were trained for $30 \mathrm{~min}$ a day in Coulbourn (Allentown, PA) operant chambers to lever press for 45-mg food pellets. The training started with continuous reinforcement (FR1), gradually increasing to a fixed ratio schedule of 15. Rats were then trained until a stable baseline response rate was reached $( \pm 10 \%$ of mean for 3 consecutive days). Once stable baselines were achieved, rats were acclimated for 3 days to a split schedule in which a 10-min baseline session was followed by a timeout period of $15 \mathrm{~min}$ followed by a 20 -min test session. This schedule permitted an assessment of daily stable baselines (10-min session) prior to any drug administration. There was no limit to the number of pellets that could be earned in either the 10- or 20-min sessions.
Conditioned Place Aversion. The apparatus and procedure have been described in detail previously (Hand et al. 1988; Schulteis et al. 1994). Briefly, the apparatus consisted of three rectangular boxes $(40 \times 33 \times 34 \mathrm{~cm})$ spaced at $120^{\circ}$ angles and all accessible from a triangular central compartment. Distinctive visual and tactile cues distinguished the three compartments (for details, see Stinus et al. 1990). Each compartment was equipped with photocells to allow automatic detection and recording of an animal's position at all times with a computer. The apparatus was located in a sound-attenuated testing room, with white noise $(75 \mathrm{~dB})$ to further mask external noise, and illuminated by three $15 \mathrm{~W}$ red lights located $1.5 \mathrm{~m}$ above each compartment.

The experimental protocol consisted of three distinct phases: a preconditioning phase, a conditioning phase, and a testing phase. In the preconditioning phase (3 days post-pellet implantation), animals were placed in the central triangular compartment and allowed to explore the apparatus freely for $20 \mathrm{~min}$. For each rat, the two compartments with the most similar time allotments were randomly paired either with a dose of naloxone $(7.5-120 \mu \mathrm{g} / \mathrm{kg})$ or vehicle. The third compartment was not paired with an injection. The unassigned compartment could be either the most or least preferred of the three. This selection and pairing procedure allowed for a minimization of the imbalance in time spent in the naloxone- versus vehicle-paired compartments. Importantly, after the compartment assignments were completed, there were no significant differences between time spent in the naloxone-paired, vehiclepaired, or neutral compartments during the preconditioning phase for any group in any experiment.

In the conditioning phase, rats received injections of vehicle on days 4,6 , and 8 post-pellet implantation prior to being confined to their preselected vehiclepaired compartment for $20 \mathrm{~min}$. On days 5, 7, and 9 post-pellet implantation, rats received one of several doses of naloxone $(7.5-120 \mu \mathrm{g} / \mathrm{kg})$ immediately prior to confinement in the naloxone-paired compartment for $20 \mathrm{~min}$. The testing phase consisted of a 20-min free exploration of the entire apparatus on day 10 post-pellet implantation. The difference in time spent on the naloxone-paired side during the testing phase and the time spent in the same compartment during the preconditioning phase served as an index of place aversion (aversion score $=$ postconditioning minus preconditioning time spent in naloxone-paired compartment).

Data Analysis. Data for all experiments were analyzed by analysis of variance (ANOVA), using either a single-factor (Experiment 2) or a mixed design two-factor (Experiments 1,3,4) model as appropriate. Post-hoc analyses consisted of comparisons of simple main effects using the Bonferroni correction to maintain a constant $p$-value of $p<.05$ for all comparisons. 


\section{Experiment 1: Clonidine Effects on Spontaneous Locomotion in Opiate-Naive Rats}

Clonidine is known to have motoric and sedative effects that could influence performance in the conditioned place aversion and conditioned suppression of responding models of opiate withdrawal. To understand the dose-effect profile and time course of this effect of clonidine and to guide design of subsequent studies with these parameters in mind, locomotor activity was assessed in standard photocell activity cages.

Experimental Design and Procedure. Rats (total 92) were given an injection of clonidine or vehicle and placed into the photocell cages for a 2 -h period to assess spontaneous locomotion in a novel environment. All testing took place at the very beginning of the dark phase to ensure a high level of baseline activity in the control groups. Each rat received only one dose of vehicle or clonidine $(3,6,12,25,50,100,200,400$, or $1,000 \mu \mathrm{g} / \mathrm{kg})$. Data were summarized and analyzed in 30-min blocks using a mixed design ANOVA with dose of clonidine as a between-subjects factor and time block as a withinsubjects factor.

In a follow-up study, a time course of clonidine action was conducted using a clonidine dose of $200 \mu \mathrm{g} /$ $\mathrm{kg}$ (this dose was used in place aversion studies). Rats (total 62) were treated with this dose of clonidine either $20 \mathrm{~min}$ or $1,3,6,9$, or $12 \mathrm{~h}$ prior to a $3-\mathrm{h}$ session in the activity cages. A control group receiving vehicle was tested 20 min after injection. Testing again took place at the beginning of the dark phase for all groups. Each rat was tested in only one condition. Data were summarized and analyzed in 1-h blocks using a mixed design ANOVA with time of clonidine pretreatment as a between-subjects factor and time block as a within-subjects factor.

Results. As shown in Table 1, clonidine dose-dependently suppressed spontaneous locomotion in opiatenaive rats. The dose-dependent nature of clonidine's ef- fect was supported by a significant main effect of clonidine dose $(\mathrm{F}[9,82]=18.90, p<.0001)$, and a significant dose $\times$ time block interaction $(F[27,246]=$ 9.18, $p<.0001)$. Post-hoc comparisons indicated that $25 \mu \mathrm{g} / \mathrm{kg}$ was the minimal effective dose of clonidine to suppress locomotion, but the effect lasted only 30 to $60 \mathrm{~min}$ at this dose. Profound suppression of activity for the entire 120-min observation period was noted at doses of $200 \mu \mathrm{g} / \mathrm{kg}$ and above.

As shown in Table 2, a dose of $200 \mu \mathrm{g} / \mathrm{kg}$ of clonidine suppressed locomotion in a time-dependent manner. The time-dependent nature of this effect was supported by a significant main effect of pretreatment time $(\mathrm{F}[6,55]=27.91, p<.0001)$ as well as a significant pretreatment time by time block interaction $(F[12,110]=6.82, p<.0001)$. Post-hoc comparisons indicated that clonidine had significant effects on locomotion with pretreatment intervals up to $6 \mathrm{~h}$, but that the effect of clonidine had dissipated by $9 h$ after administration.

\section{Experiment 2: Effect of Clonidine on Operant Responding for Food in Opiate-Naive and Morphine-Dependent Rats}

Experimental Design and Procedure. To determine the effects of clonidine alone on operant responding for food, one group of rats $(n=9)$ was tested following vehicle and 5,10, and $20 \mu \mathrm{g} / \mathrm{kg}$ doses of clonidine in a within-subjects Latin square design, with one dose being administered on each of 4 consecutive days. These doses were selected based upon previous work (Sparber and Meyer 1978) using a similar operant paradigm to assess opiate withdrawal. After a 10-min baseline, each rat received one of the four doses on a given day, then were returned to the home cage for $15 \mathrm{~min}$. They were then given a SC injection of saline, and resumed a 20-min session in the operant boxes.

To evaluate the effects of clonidine on unconditioned suppression of operant responding by naloxone,

Table 1. Effects of Clonidine on Spontaneous Locomotor Activity as a Function of Dose

\begin{tabular}{lrccccc}
\hline & $\boldsymbol{n}$ & Min 0-30 & Min 30-60 & Min 60-90 & Min 90-120 & Total Activity \\
\hline Vehicle & 10 & $505.1 \pm 65.6$ & $191.3 \pm 28.3$ & $239.3 \pm 42.6$ & $192.0 \pm 37.4$ & $1128 \pm 148$ \\
$3 \mu \mathrm{g} / \mathrm{kg}$ & 9 & $532.6 \pm 40.5$ & $248.3 \pm 35.0$ & $223.0 \pm 28.0$ & $194.4 \pm 26.0$ & $1198 \pm 98$ \\
$6 \mu \mathrm{g} / \mathrm{kg}$ & 10 & $470.0 \pm 26.1$ & $251.3 \pm 21.8$ & $250.3 \pm 29.1$ & $179.2 \pm 35.9$ & $1151 \pm 85$ \\
$12 \mu \mathrm{g} / \mathrm{kg}$ & 10 & $379.0 \pm 23.0$ & $175.4 \pm 18.7$ & $207.0 \pm 25.0$ & $219.4 \pm 39.5$ & $981 \pm 65$ \\
$25 \mu \mathrm{g} / \mathrm{kg}$ & 10 & $248.8 \pm 30.4$ & $157.7 \pm 18.7$ & $188.5 \pm 33.1$ & $178.5 \pm 21.0$ & $773 \pm 83^{*}$ \\
$50 \mu \mathrm{g} / \mathrm{kg}$ & 10 & $172.2 \pm 18.5$ & $107.8 \pm 16.5$ & $138.7 \pm 21.3$ & $136.0 \pm 19.9$ & $555 \pm 58^{*}$ \\
$100 \mu \mathrm{g} / \mathrm{kg}$ & 11 & $118.9 \pm 18.5$ & $107.9 \pm 28.6$ & $122.9 \pm 25.6$ & $153.5 \pm 28.7$ & $503 \pm 87^{*}$ \\
$200 \mu \mathrm{g} / \mathrm{kg}$ & 7 & $77.5 \pm 17.8$ & $39.0 \pm 9.5$ & $32.1 \pm 5.7$ & $45.9 \pm 7.0$ & $194 \pm 21^{*}$ \\
$400 \mu \mathrm{g} / \mathrm{kg}$ & 7 & $71.1 \pm 10.7$ & $104.1 \pm 27.9$ & $78.4 \pm 27.2$ & $21.4 \pm 6.3$ & $22.9 \pm 7.7$ \\
$1000 \mu \mathrm{gg} / \mathrm{kg}$ & 8 & $169.8 \pm 24.5$ & $60.8 \pm 21.3$ & $37.8 \pm 12.7$ & 22.9 \\
\hline
\end{tabular}


Table 2. Effects of Clonidine $(200 \mu \mathrm{g} / \mathrm{kg})$ on Spontaneous Locomotor Activity as a Function of Pretreatment Time

\begin{tabular}{lrrrrr}
\hline & $\boldsymbol{n}$ & Min 0-60 & Min 60-120 & Min 120-180 & Total Activity \\
\hline $\begin{array}{l}\text { No clonidine } \\
\begin{array}{l}\text { Clonidine } \\
\quad \text { pretreatment: }\end{array}\end{array}$ & 11 & $758.5 \pm 50.4$ & $410.5 \pm 49.5$ & $409.2 \pm 76.3$ & $1578 \pm 140$ \\
& & & & & \\
20 min & 6 & $87.8 \pm 40.5$ & $49.3 \pm 13.3$ & $102.2 \pm 13.5$ & $239 \pm 35^{*}$ \\
1 h & 8 & $114.5 \pm 28.3$ & $92.2 \pm 18.4$ & $123.6 \pm 16.3$ & $330 \pm 43^{*}$ \\
3 h & 8 & $158.4 \pm 31.9$ & $123.8 \pm 21.7$ & $184.3 \pm 28.5$ & $466 \pm 48^{*}$ \\
9 h & 10 & $389.7 \pm 56.2$ & $201.5 \pm 24.1$ & $190.9 \pm 19.6$ & $782 \pm 86^{*}$ \\
$12 \mathrm{~h}$ & 9 & $702.3 \pm 57.8$ & $301.4 \pm 36.3$ & $226.4 \pm 47.2$ & $1230 \pm 102$ \\
& 10 & $656.6 \pm 47.8$ & $385.5 \pm 50.3$ & $418.0 \pm 77.1$ & $1460 \pm 123$ \\
\hline
\end{tabular}

Data represent mean \pm SE number of photocell counts.

${ }^{*} p<.01$ vs. vehicle control using Bonferroni correction.

a separate group of rats $(n=8)$ was trained to stable baseline criteria as described above, then implanted with morphine pellets and allowed to recover for 3 days. Beginning 3 days after pellet implantation, rats were acclimated to the $10 \mathrm{~min} / 20 \mathrm{~min}$ split schedule procedure described above. Beginning on day 6 post-pellet implantation, rats were given a $10-\mathrm{min}$ session, followed immediately by an injection of vehicle or clonidine (5, 10 , or $20 \mu \mathrm{g} / \mathrm{kg}$ ). Fifteen min after this first injection, rats were treated with a $25 \mu \mathrm{g} / \mathrm{kg}$ dose of naloxone and then immediately placed back in the operant chambers for a 20-min test session. Once again, vehicle and clonidine doses were administered using a within-subjects Latin square design, with one dose being administered on each of 4 consecutive days.

All operant data were summarized as the percentage of baseline, with responding during the 20-min test session expressed as a percentage of the response rate during the 10-min baseline session on that same day. Data were analyzed using a one-factor withinsubjects ANOVA design.

Results. As shown in Figure 1a, clonidine dose-dependently disrupted responding by itself in opiate-naive rats $(\mathrm{F}[3,24]=13.95, p<.0001)$. Both the 10 and $20 \mu \mathrm{g} / \mathrm{kg}$ doses of clonidine modestly, but significantly, suppressed responding. Importantly, analysis of the 10-min baseline data revealed no significant carry-over effects of any dose of clonidine administered on the prior day (data not shown).

As shown in Figure 1b, clonidine dose-dependently alleviated the suppression of responding produced by naloxone-precipitated withdrawal $(\mathrm{F}[3,21]=4.905$, $p<.01)$. Doses of 5 and $10 \mu \mathrm{g} / \mathrm{kg}$ of clonidine significantly increased responding in naloxone-treated rats. This was seen despite the fact that $10 \mu \mathrm{g} / \mathrm{kg}$ of clonidine could suppress responding on its own (Figure 1a). However, a clonidine dose of $20 \mu \mathrm{g} / \mathrm{kg}$ did not significantly attenuate withdrawal, most likely because of the more pronounced response-suppressing effects of this dose by itself (Figure 1a). Based upon these re- sults, a dose of $10 \mu \mathrm{g} / \mathrm{kg}$ was chosen as the most efficacious dose for alleviating naloxone-precipitated withdrawal, and this dose was used in the subsequent conditioning studies.

\section{Experiment 3: Effects of Clonidine on Acquisition and Expression of Conditioned Suppression of Operant Responding During Opiate Withdrawal}

Experimental Design and Procedure. Rats (total 27) were trained to respond on an FR-15 schedule for food reward, implanted with morphine pellets, allowed to recover for 3 days and then acclimated to the 10-min/ 20-min split schedule described above. Beginning on day 6 post-pellet implantation, rats were randomly assigned to one of three groups (control, acquisition, expression) and began conditioning trials. Following a 10-min baseline session, rats received either an SC injection of saline (control and expression groups) or $10 \mu \mathrm{g} /$ $\mathrm{kg}$ clonidine (acquisition group) and placed back in the home cage for $15 \mathrm{~min}$. All rats then received a $25 \mu \mathrm{g} / \mathrm{kg}$ SC injection of naloxone, and were placed in the operant box for a 20-min conditioning session in which a compound conditioned stimulus (CS) consisting of a tone $(7 \mathrm{kHz}, 85 \mathrm{~dB})$ and an odor (anise extract) were paired with naloxone-precipitated withdrawal. Rats received two such conditioning sessions on consecutive days, followed by a baseline day in which all rats received only saline injections in place of both clonidine and naloxone, followed by two more consecutive conditioning sessions.

Twenty-four $\mathrm{h}$ after the final conditioning session, the control and acquisition groups both received a SC saline injection after their 10-min baseline session. After waiting $15 \mathrm{~min}$ in the home cage, they were given another SC injection of saline and put back into the boxes for $20 \mathrm{~min}$ with the tone and odor being presented as in the conditioning phase. The expression group received a $10 \mu \mathrm{g} / \mathrm{kg}$ dose of clonidine, $15 \mathrm{~min}$ in home cage, and a 20-min session with the presentation of the CS just as 


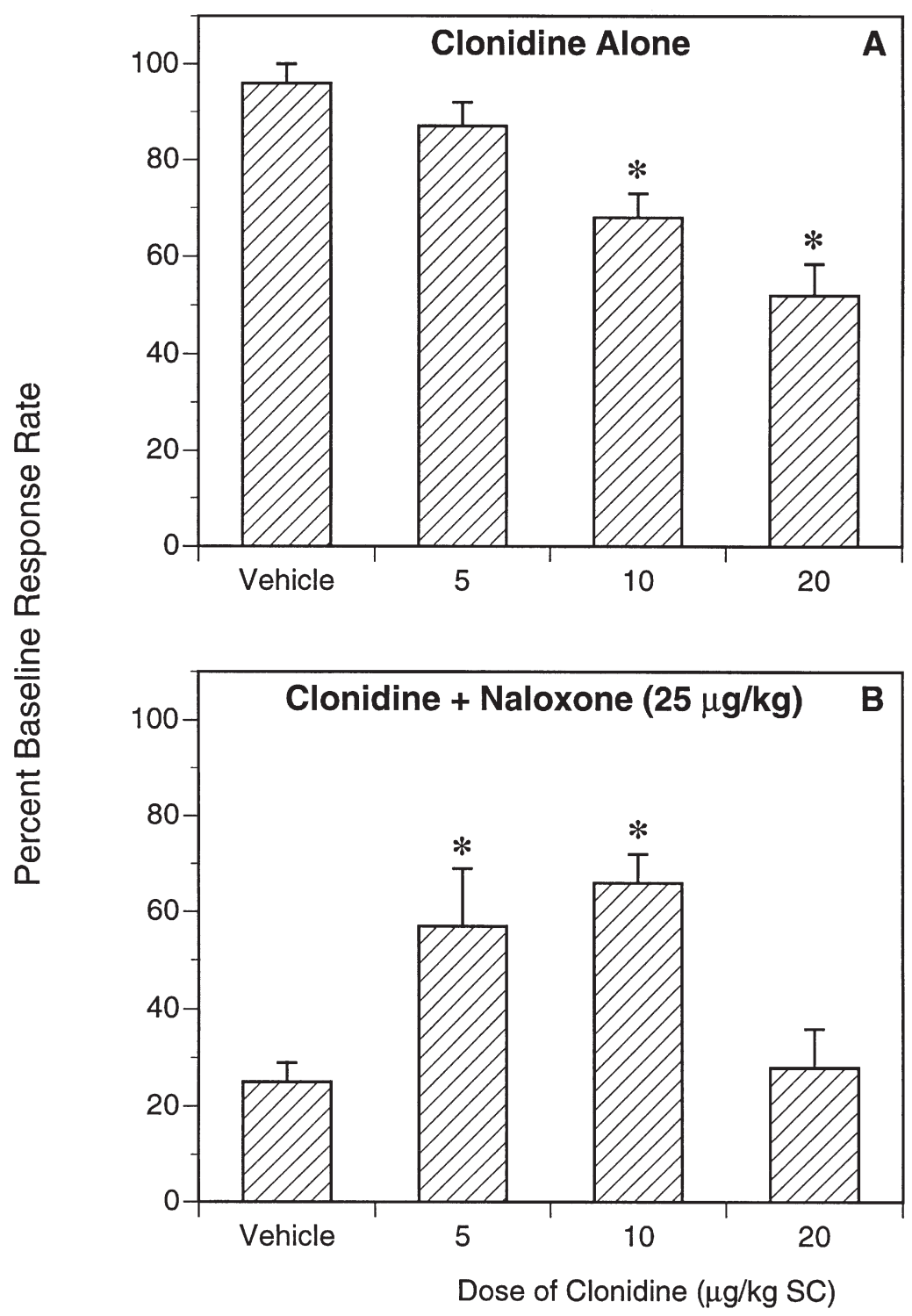

Figure 1. Clonidine administered subcutaneously (SC) dose-dependently suppresses operant responding for food (panel A) in opiate-naive rats, but nonetheless attenuates naloxone-precipitated $(25 \mu \mathrm{g} / \mathrm{kg})$ suppression of responding in opiate-dependent rats. $\left({ }^{*} p<.05\right.$ vs. corresponding vehicle control condition). the other two groups. Each animal received a postconditioning baseline day $24 \mathrm{~h}$ after the CS test day.

The average of the baseline day between the second and third conditioning trials and the postconditioning baseline served as a drug-free, CS-Free baseline response rate against which response rates during the CS test session could be compared. Data were analyzed as a mixed design ANOVA with treatment group (control, acquisition, expression) as a between-subjects factor and session (preconditioning baseline vs. postconditioning test) as a within-subjects factor.

Results. The overall ANOVA revealed a significant effect of group $(\mathrm{F}[2,20]=13.388, p<.001)$, day $(\mathrm{F}[1,20]=$ $113.195, p<.0001)$, and group by day interaction $(\mathrm{F}[1$, $20]=14.169, p<.0001)$. As shown in Figure 2, the control group showed a significant difference between baseline days and the CS test day, indicating the development of conditioned suppression of responding (con- ditioned withdrawal). The acquisition group, which received clonidine prior to each conditioning session and saline prior to the test session, showed no incidence of conditioned withdrawal. In contrast, the expression group, which received clonidine only prior to the test session, continued to demonstrate a significant conditioned withdrawal.

\section{Experiment 4: Effect of Clonidine on Conditioned Place Aversion Produced by Naloxone in Morphine- Dependent Rats}

Experimental Design and Procedure. In an initial study to determine the clonidine dose-effect function, all rats (total 92) received a dose of $7.5 \mu \mathrm{g} / \mathrm{kg}$ of naloxone on the days of withdrawal. Separate groups of rats received either vehicle or one dose of clonidine $(6,12.5$, $25,50,100,200$, or $400 \mu \mathrm{g} / \mathrm{kg}) 20 \mathrm{~min}$ prior to each 


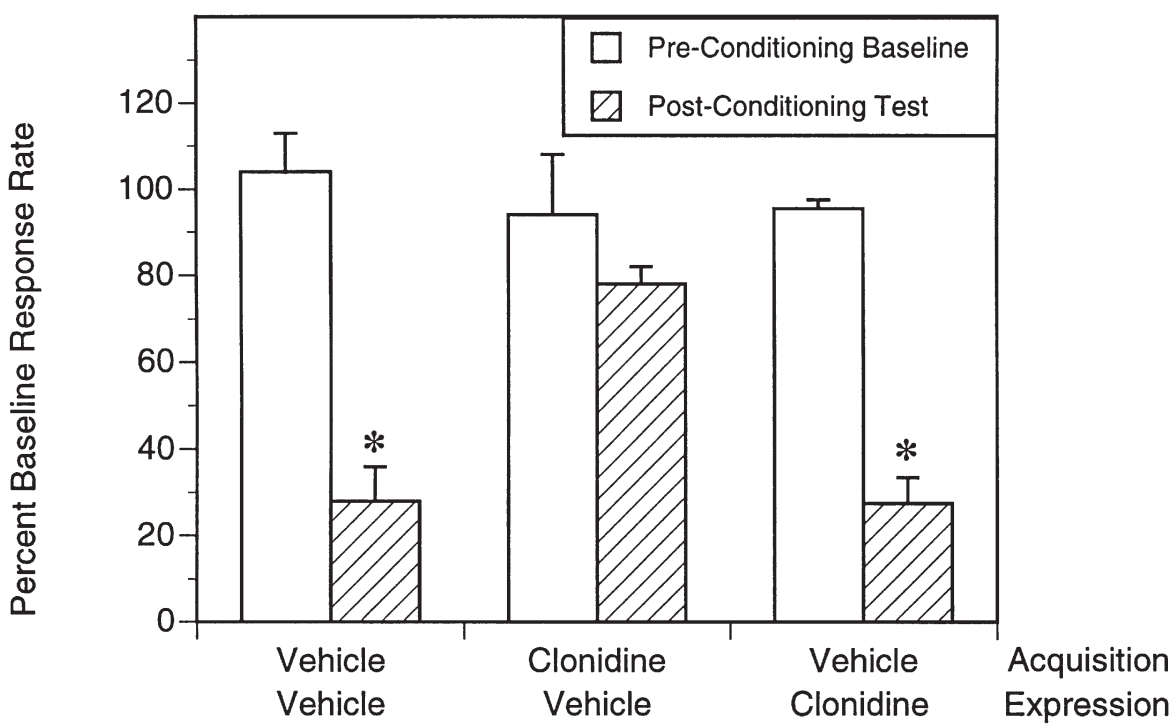

Figure 2. Clonidine (10 $\mu \mathrm{g} / \mathrm{kg})$ administered subcutaneously (SC) blocks the acquisition but not expression of conditioned suppression of operant responding. Animals treated with vehicle during acquisition (prior to each naloxone-precipitated withdrawal [25 $\mu \mathrm{g} / \mathrm{kg} \mathrm{SC}$ ] conditioning session) and expression (prior to the postconditioning test session) of conditioned opiate withdrawal showed a significant reduction in response rates upon presentation of the naloxone-paired tone/odor stimulus by itself. This conditioned withdrawal response was eliminated in animals treated with clonidine during acquisition. By contrast, animals treated with clonidine only prior to the postconditioning session (expression) continued to show a significant conditioned withdrawal response $\left({ }^{*} p<.05\right.$ vs. corresponding preconditioning baseline).

naloxone injection; each rat received the same dose of clonidine on each naloxone-conditioning day. Data were analyzed by mixed design ANOVA, with clonidine dose as a between-subjects factor and conditioning phase (pre or postconditioning) as the within-subjects factor.

A follow-up study was designed to determine whether clonidine was as effective in blocking the expression of an established conditioned place aversion as it was in blocking acquisition of the aversion. Rats $(n=50)$ were divided into three groups: (1) vehicle controls receiving saline injections prior to each conditioning session and the postconditioning test session; (2) the acquisition group receiving clonidine $(200 \mu \mathrm{g} / \mathrm{kg}) 3 \mathrm{~h}$ prior to each naloxone-paired $(7.5 \mu \mathrm{g} / \mathrm{kg})$ conditioning session and saline prior to the postconditioning test session; and (3) the expression group receiving saline prior to each conditioning session but clonidine $3 \mathrm{~h}$ prior to the test session. The $200 \mu \mathrm{g} / \mathrm{kg}$ dose was chosen as the minimum dose which completely reversed the acquisition of conditioned place aversion (see Figure 3 ). The $3 \mathrm{~h}$ time point was chosen to limit the overt activity-suppressing effects of clonidine at this high dose (see Experiment 1), but it was deemed acceptable, because prior work (Schulteis et al. 1997b; Stinus, unpublished observations) had indicated that $200 \mu \mathrm{g} / \mathrm{kg}$ of clonidine administered as long as 6-h prior to each conditioning session blocked the acquisition of conditioned place aversions. To confirm this in the present report, rats in the acquisition group also were treated with clonidine $3 \mathrm{~h}$ prior to the conditioning sessions. Data were analyzed by mixed design ANOVA with drug condition as the between-subjects factor and conditioning phase (pre or postconditioning) as the within-subjects factor.

Results. As shown in Figure 3, clonidine administered prior to each naloxone administration during conditioning dose-dependently attenuated the conditioned place aversion precipitated by naloxone in morphine-dependent rats. The dose-dependent nature of the effect was supported by a significant main effect of clonidine dose $(\mathrm{F}[7,84]=3.04, p<.01)$, and a significant clonidine dose $\times$ conditioning phase interaction $(F[7,84]=5.12, p<.0001)$. Visual inspection of the data (Figure 3) suggested a partial attenuation of place aversion at doses of 25 to $100 \mu \mathrm{g} / \mathrm{kg}$ of clonidine, with a complete reversal at doses of 200 to $400 \mu \mathrm{g} / \mathrm{kg}$. This impression was supported by post-hoc comparisons that indicated significant differences in time spent in the naloxone-paired compartment pre and postconditioning only for groups treated with vehicle, $6 \mu \mathrm{g} / \mathrm{kg}$ or 12.5 $\mu \mathrm{g} / \mathrm{kg}$ of clonidine, indicating a significant reduction in place aversion by doses of clonidine as low as $25 \mu \mathrm{g} / \mathrm{kg}$.

The data on clonidine effects on acquisition and expression of conditioned place aversions are presented graphically in Figure 4. The overall ANOVA revealed a significant main effect of drug condition $(\mathrm{F}[2,47]=$ 23.72, $p<.0001)$ and a significant drug conditioning by conditioning phase interaction $(\mathrm{F}[1,47]=32.66, p<$ .0001). Follow-up comparison of the vehicle and acqui- 


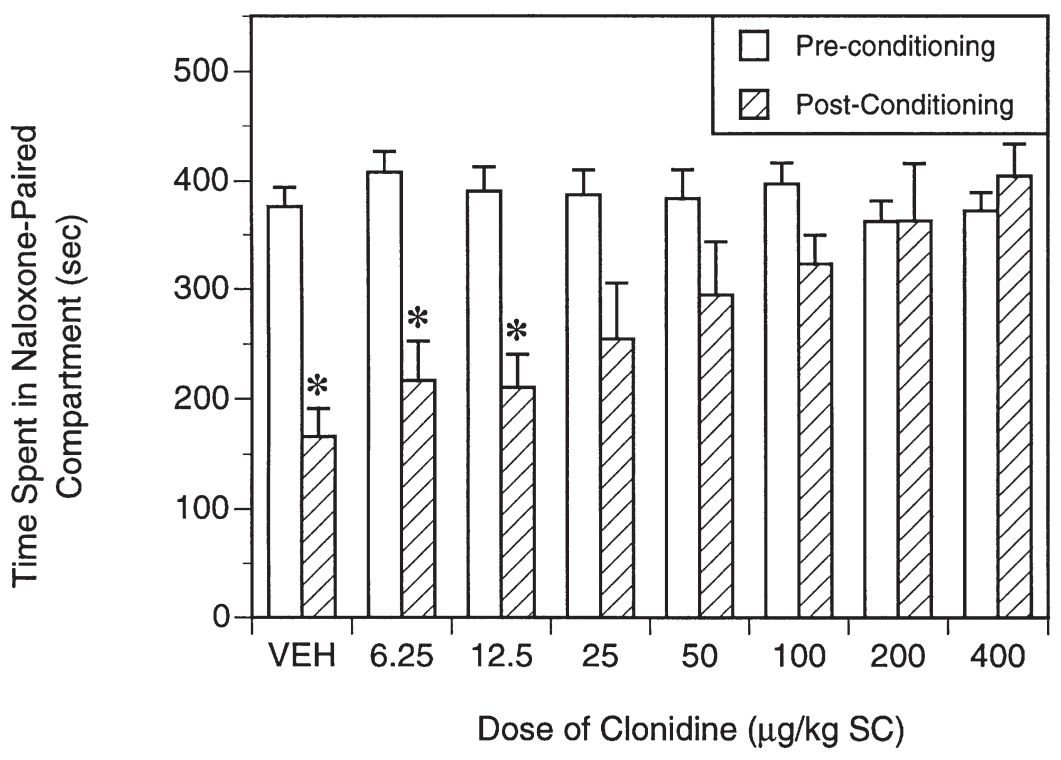

Figure 3. Clonidine administered subcutaneously (SC) dose-dependently reverses the conditioned place aversion produced by naloxone-precipitated $(7.5 \mu \mathrm{g} / \mathrm{kg} \mathrm{SC})$ opiate withdrawal. A significant place aversion $\left({ }^{*} p<\right.$ .05 with Bonferroni correction) was seen in vehicle-treated controls and in groups receiving the two lowest doses of clonidine (6.25$12.5 \mu \mathrm{g} / \mathrm{kg}$ ). However, at doses of $25 \mu \mathrm{g} / \mathrm{kg}$ clonidine and higher, the difference in time spent in the naloxone-paired compartment preconditioning and postconditioning was no longer significant. sition groups revealed a complete reversal of place aversion in the acquisition group as supported by a significant main effect of drug condition $(\mathrm{F}[1,37]=21.71$, $p<.0001)$ and a significant condition $\times$ conditioning phase interaction $(\mathrm{F}[1,37]=26.40, p<.0001)$. Thus, clonidine was completely effective in attenuating acquisition of conditioned place aversion when administered $3 \mathrm{~h}$ prior to each conditioning session, confirming our earlier results (Schulteis et al. 1997b; Stinus, unpublished observations).

Follow-up comparison of the vehicle and expression groups also revealed a significant main effect of drug condition $(\mathrm{F}[1,38]=9.91, p<.005)$ and a significant drug $\times$ conditioning phase interaction $(F[1,38]=20.91$, $p<.0001$ ); however, as shown in Figure 4, these significant overall effects were accounted for not by an attenu-

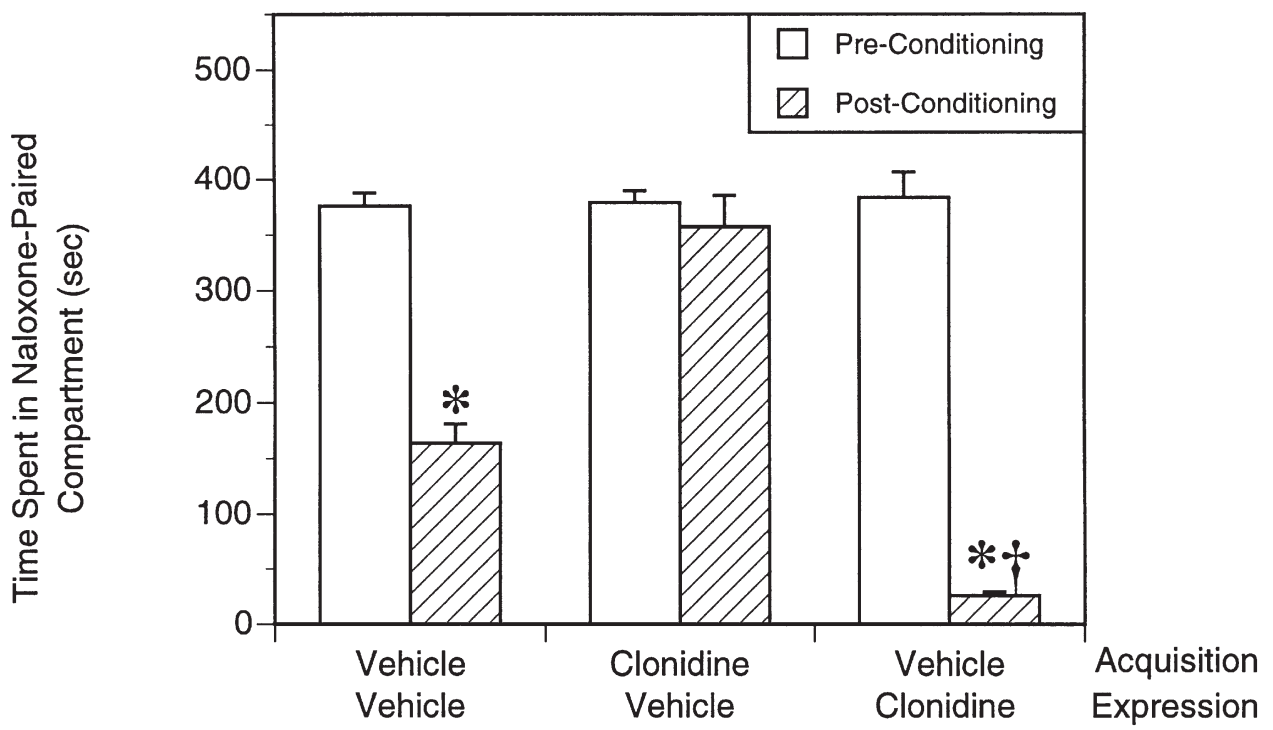

Figure 4. Clonidine $(200 \mu \mathrm{g} / \mathrm{kg})$ administered subcutaneously (SC) blocks the acquisition but not expression of conditioned place aversion produced by naloxone-precipitated $(7.5 \mu \mathrm{g} / \mathrm{kg} \mathrm{SC})$ opiate withdrawal. Animals treated with vehicle during acquisition (prior to each conditioning session) and expression (prior to the postconditioning test session) showed a significant place aversion, and this aversion was eliminated in animals treated with clonidine during acquisition. By contrast, animals treated with clonidine only prior to the postconditioning session (expression) showed a significant potentiation of place aversion $\left({ }^{*} p<.05\right.$ vs. corresponding preconditioning baseline; $t p<.05$ vs. vehicle-vehicle condition). 
ation of place aversion by clonidine, but by a significant increase in the place aversion observed in the expression group relative to vehicle controls.

\section{DISCUSSION}

The principal finding of the current set of studies is that clonidine is effective in attenuating conditioned opiate withdrawal only when administered prior to each conditioning trial. When clonidine was administered prior to the test session, after conditioning had already taken place, it was found to be ineffective in altering the conditioned withdrawal response. It is well known that manipulation of noradrenergic systems by a variety of agents, including clonidine, can modulate learning and memory storage processes to produce either enhancement or impairment of memory, depending upon strength of training, dose of drug administered, and their interaction (for example, see Martinez et al. 1991; McGaugh 1992). Thus, it is unclear whether the efficacy of clonidine in blocking acquisition of the conditioned responses was as a result of its ability to attenuate withdrawal symptomatology directly, or attributable to the modulation of associative learning processes by the drug. Regardless, the main conclusion that clonidine cannot effectively attenuate previously established conditioned responses is unaltered.

Low doses (10-50 $\mu \mathrm{g} / \mathrm{kg})$ of clonidine were effective in attenuating both the conditioned suppression of responding and conditioned place aversions produced by naloxone in morphine pellet-implanted rats. Importantly, previous work in our laboratory has established that three pairings of naloxone $(8-60 \mu \mathrm{g} / \mathrm{kg} \mathrm{SC})$ with a distinct environment does not produce a conditioned place aversion in opiate-naive rats under experimental conditions identical to those employed herein (Schulteis et al. 1994). Therefore, the effects of clonidine in the current study can be interpreted as an interaction with the ability of naloxone to induce aversive stimulus effects in opiate-dependent rats, and not a nonspecific interaction between clonidine and naloxone on place conditioning behavior independent of morphine exposure history.

Kosten (1994) has suggested that the intrinsic reinforcing properties of clonidine itself may be required to overcome the aversive consequences of opiate withdrawal as measured in the place aversion paradigm. The minimal doses of clonidine found to be effective in the current study, however, are below those reported to produce a conditioned place preference (Cervo et al. 1993; Tierney et al. 1988). Our findings are in agreement with those of Nader and van der Kooy (1996), who found that $50 \mu \mathrm{g} / \mathrm{kg}$ of clonidine could attenuate conditioned place aversions produced by naloxone-precipitated opiate withdrawal without producing any conditioned preferences by itself in opiate-dependent, nonwithdrawn rats. Nonetheless, it is possible that the complete reversal of place aversion seen with 100 to $400 \mu \mathrm{g} / \mathrm{kg}$ doses of clonidine was dependent, at least in part, on the ability of these doses to produce a place preference (Cervo et al. 1993; Nader and van der Kooy 1996; Tierney et al. 1988).

The results of the current study also confirm a wellknown side effect of clonidine and other $\alpha_{2}$ agonists: suppression of activity. In experiments 1 and 2, clonidine was found to dose-dependently suppress both spontaneous locomotion and operant responding for food in opiate-naive animals with relatively equal potency $(10-25 \mu \mathrm{g} / \mathrm{kg}$ in both studies). The dose-effect function obtained using the operant paradigm is in close agreement with earlier reports (McCleary and Leander 1981; Sparber and Meyer 1978). We could argue, therefore, that clonidine was ineffective in attenuating expression of conditioned opiate withdrawal when administered prior to the test sessions because of its intrinsic sedative and response-disruptive effects. However, arguing against this interpretation is the finding that the same dose of clonidine that fails to alter expression of conditioned suppression of operant responding in experiment $3(10 \mu \mathrm{g} / \mathrm{kg})$ was able to attenuate unconditioned suppression of responding produced by naloxone in morphine-dependent rats in experiment 2. Animals treated with $10 \mu \mathrm{g} / \mathrm{kg}$ of clonidine and $25 \mu \mathrm{g} / \mathrm{kg}$ of naloxone were able to respond at $70 \%$ of their baseline rate, a rate equivalent to that seen following administration of clonidine by itself to opiate-naive rats. These data suggest the more likely interpretation that clonidine is ineffective in blocking expression of conditioned withdrawal not because of its inherent sedative properties, but because of its inability to alter a conditioned response once it has been established.

The activity-suppressing effects of clonidine may have contributed, however, to the enhanced place aversion seen when this drug was administered $3 \mathrm{~h}$ prior to the test for expression of conditioned withdrawal. As shown in experiment 1 , clonidine $(200 \mu \mathrm{g} / \mathrm{kg})$ administered $3 \mathrm{~h}$ prior to assessment of locomotor activity induced a robust, although far from complete, suppression of locomotion (see Table 2). Thus, reduced activity may have contributed to a greater apparent aversion of the naloxone-paired compartment, because the rats were less likely to locomote into the aversive compartment from their preferred compartments. However, the effects on locomotion cannot account for the failure to reverse the previously established conditioned aversion. A nonspecific motoric deficit would not be expected to reduce activity selectively in a single compartment in the absence of some motivational impetus to avoid that compartment. An alternative explanation for the enhanced aversion seen when clonidine was administered prior to the test for expression may be a facili- 
tated retrieval of the conditioned association produced by clonidine through action on noradrenergic systems that modulate memory storage processes (e.g., Martinez et al. 1991; McGaugh 1992).

The current data in rats confirm earlier preclinical reports of Kosten (1994) and Nader and van der Kooy (1996) in suggesting that affective signs of opiate withdrawal can be blocked by clonidine, as can acquisition of conditioned opiate withdrawal. However, as discussed earlier, although data from the clinical literature support some reductions in subjective/affective components of withdrawal, these symptoms seem less effectively blocked by clonidine than somatic signs. Many current theories of addiction favor a role of affective withdrawal symptomatology and conditioned reinforcement processes in maintaining addiction and promoting relapse after periods of abstention (Childress et al. 1994; Jasinski et al. 1985; O'Brien et al. 1976, 1993; Ramsay and Woods 1997; Schulteis and Koob 1996; Schulteis et al. 1997a; Wikler 1973). The inability of clonidine to attenuate effectively either unconditioned or conditioned affective withdrawal symptoms emphasizes a potentially significant limitation in the clinical utility of this and related $\alpha_{2}$-compounds (e.g., lofexidine, Kahn et al. 1997; Washton et al. 1983) in facilitating complete detoxification and preventing relapse.

However, this problem may not be unique to $\alpha_{2}$-noradrenergic agents, but would likely apply to most pharmacotherapeutic treatments. For example, the dopaminergic receptor blocker pimozide can alter the motivational properties of food and cocaine as reinforcers in the acquisition of conditioned behaviors, but fails to affect expression of conditioned responses to cocaine or food once established (e.g., Beninger and Herz 1986; Horvitz and Ettenberg 1991). Within the context of opiate withdrawal, if a drug lessens the impact of the unconditioned stimulus (UCS, removal of opiates from the receptors) and consequently the emergence of the unconditioned response (UCR, somatic and affective signs of withdrawal), it would be expected to impair the ability of environmental cues to become associated with the UCS through processes of classical conditioning ( $\mathrm{O}^{\prime}$ Brien et al. 1976, 1993; Ramsay and Woods 1997). Therefore, it could be efficacious in preventing acquisition of conditioned drug withdrawal (conditioned response, CR). However, once the associative process has taken place, the conditioned stimuli (CS) may evoke the CR independent of the UCS-UCR pathway, and thereby, expression of conditioned withdrawal may be resistant to the same drugs that can block the unconditioned withdrawal response. Thus, in developing effective treatment strategies, it is important to recognize that the expression of the conditioned response may involve a separate neurobiological substrate more closely tied to memory processing systems than systems directly subserving opiate withdrawal.

\section{ACKNOWLEDGMENTS}

This is publication number 11123-NP from the Scripps Research Institute. Work presented herein was supported in part by grants from the National Institute on Drug Abuse (DA0 4043 to GFK, DA10475 to GS), and grants from the Université de Bordeaux 2, the CNRS, and the Fondation Pour La Recherche Médicale, Région Aquitaine Pole Médicament (all to LS).

\section{REFERENCES}

Baldwin HR, Koob GF (1993): Rapid induction of conditioned opiate withdrawal in the rat. Neuropsychopharmacology 8:15-21

Beninger RJ, Herz RS (1986): Pimozide blocks establishment but not expression of cocaine-produced environmentspecific conditioning. Life Sic 38:1425-31

Britton, KT, Svensson T, Schwartz J, Bloom FE, Koob G (1984): Dorsal noradrenergic bundle lesions fail to alter opiate withdrawal or suppression of opiate withdrawal by clonidine. Life Sci 34:133-139

Cervo L, Rossi C, Samanin R (1993): Clonidine-induced place preference is mediated by $\alpha_{2}$-adrenoceptors outside the locus coeruleus. Eur J Pharmacol 238:201-207

Charney DS, Sternberg DE, Kleber HD, Heninger MD, Redmond DE Jr (1981): The clinical use of clonidine in abrupt withdrawal from methadone: Effects on blood pressure and specific signs and symptoms. Arch Gen Psychiat 38:1273-1277

Childress AR, Ehrman R, McLellan AT, MacRae J, Natale M, O'Brien CP (1994): Can induced moods trigger drugrelated response in opiate abuse patients? J Subst Abuse Treat 11:17-23

Coupar IM (1992): Effect of $\alpha_{2}$-adrenoceptor agonists in the expression of morphine-withdrawal in rats. NaunynSchmiedeberg's Arch Pharmacol 345:553-557

Cuthill JD, Beroniade MD, Salvatori VA, Viguié F (1990): Evaluation of clonidine suppression of opiate withdrawal reactions: A multidisciplinary approach. Can J Psychiat 35:377-82

Dawe S, Gray JA (1995): Craving and drug reward: A comparison of methadone and clonidine in detoxifying opiate addicts. Drug Alcohol Depend 39:207-212

Gold LH, Stinus L, Inturrisi CE, Koob GF (1994): Prolonged tolerance, dependence, and abstinence following subcutaneous morphine pellet implantation in the rat. Eur J Pharmacol 253:45-51

Gold MS, Redmond DE Jr, Kleber HD (1978): Clonidine blocks acute opiate-withdrawal symptoms. Lancet 2:599-602

Hand TH, Koob GF, Stinus L, Le Moal M (1988): Aversive properties of opioid receptor blockade: Evidence for exclusively central mediation in naive and morphinedependent rats. Brain Res 474:364-368

Horvitz JC, Ettenberg A (1991): Conditioned incentive properties of a food-paired conditioned stimulus remain intact during dopamine receptor blockade. Behav Neurosci 105:536-541

Janiri L, Mannelli P, Persico AM, Serretti A, Tempesta E 
(1994): Opiate detoxification of methadone maintenance patients using lefetamine, clonidine, and buprenorphine. Drug Alcohol Depend 36:139-145

Jaffe JH (1990): Drug addiction and drug abuse. In Goodman-Gilman A, Rall TW, Nies AS, Taylor P (eds), The Pharmacological Basis of Therapeutics, New York, Pergamon Press, pp 522-573

Jasinski DR, Johnson RE, Kocher TR (1985): Clonidine in morphine withdrawal: Differential effects on signs and symptoms. Arch Gen Psychiat 42:1063-1066

Kahn A, Mumford JP, Rogers GA, Beckford H (1997): Double-blind study of lofexidine and clonidine in the detoxification of opiate addicts in hospital. Drug Alcohol Depend 44:57-61

Kantak KM, Miczek KA (1988): Social, motor, and autonomic signs of morphine withdrawal: Differential sensitivities to catecholaminergic drugs in mice. Psychopharmacology 96:468-476

Kasvikis Y, Bradley, Gossop M, Griffits P, Marks I (1990): Clonidine versus long- and short-methadone-aided withdrawal from opiates: An uncontrolled comparison. Int J Addict 25:1169-1174

Katz JL (1986): Effects of clonidine and morphine on opioid withdrawal in rhesus monkeys. Psychopharmacology 88:392-397

Kelsey JE, Aranow JS, Matthews RT (1990): Context-specific morphine withdrawal in rats: Duration and effects of clonidine. Behav Neurosci 104:704-710

Koob GF, Wall TL, Bloom FE (1989): Nucleus accumbens as a substrate for the aversive stimulus effects of opiate withdrawal. Psychopharmacology 98:530-534

Kosten TA (1994): Clonidine attenuates conditioned aversion produced by naloxone-precipitated opiate withdrawal. Eur J Pharmacol 254:59-63

Maldonado R, Stinus L, Koob GF (1996): Molecular Mechanisms of Opiate Withdrawal. New York, Springer.

Martinez JL Jr, Schulteis G, Weinberger SB (1991): How to increase and decrease the strength of memory traces: The effects of drugs and hormones. In Martinez JL Jr, Kesner RP (eds), Learning and Memory: A Biological View, New York, Academic Press, pp 149-198

McCleary PE, Leander JD (1981): Clonidine analgesia and suppression of operant responding: Dissociation of mechanism. Eur J Pharmacol 69:63-69

McGaugh JL (1992): Affect, neuromodulatory systems, and memory storage. In Christianson S-A (ed), The Handbook of Emotion and Memory: Research and Theory, Hillsdale, NJ, Lawrence Erlbaum Associates, pp 245-268

Nader K, van der Kooy D (1996): Clonidine antagonizes the aversive effects of opiate withdrawal and the rewarding effects of morphine only in opiate withdrawn rats. Behav Neurosci 110:389-400

O'Brien CP, Testa T, O'Brien TJ, Greenstein R (1976): Conditioning in human opiate addicts. Pav J Biol Sci 11:195-203

O'Brien CP, Childress AR, McLellan AT, Ehrman R (1993): Developing treatments that address classical conditioning. NIDA Res Monogr 135:71-91

Ramsay DS, Woods SJ (1997): Biological consequences of drug administration: Implications for acute and chronic tolerance. Psychol Rev 1997 104:170-193

Schulteis G, Gold LH, Koob GF (1997a): Preclinical behavioral models for addressing unmet needs in opiate addiction. Sem Neurosci 9:94-109

Schulteis G, Koob GF (1996): Reinforcement processes in opiate addiction: A homeostatic model. Neurochem Res 21:1437-1454

Schulteis G, Markou A, Gold LH, Stinus L, Koob GF (1994): Relative sensitivity to naloxone of multiple indices of opiate withdrawal: A quantitative dose-response analysis. J Pharmacol Exp Ther 271:1391-1398

Schulteis G, Stinus L, Risbrough V, Weinger MB, Koob GF (1997b): Clonidine blocks acquisition but not expression of conditioned opiate withdrawal. Soc Neurosci Abstr 23:528

Sparber SB, Meyer DR (1978): Clonidine antagonizes naloxone-induced suppression of conditioned behavior and body weight loss in morphine-dependent rats. Pharmacol Biochem Behav 9:319-325

Stinus L, Le Moal M, Koob GF (1990): The nucleus accumbens and amygdala as possible substrates for the aversive stimulus effects of opiate withdrawal. Neuroscience 37:767-773

Taylor JR, Elsworth JD, Garcia EJ, Grant SJ, Roth RH, Redmond DE Jr (1988): Clonidine infusions into the locus coeruleus attenuate behavioral and neurochemical changes associated with naloxone-precipitated withdrawal. Psychopharmacology 96:121-124

Tierney C, Nadaud D, Koening-Berard E, Stinus L (1988): Effects of two alpha 2 agonists, rilmenidine and clonidine, on the morphine withdrawal syndrome and their potential addictive properties in rats. Am J Cardiol 61:35D-38D

Washton AM, Resnich RB, Geyer G (1983): Opiate withdrawal using lofexidine, a clonidine analogue with fewer side effects. J Clin Psychiat 44:335-337

Wikler A (1973): Dynamics of drug dependence: Implications of a conditioning theory for research and treatment. Arch Gen Psychiat 28:611-616 